\title{
Second San Juan photoelectric astrolabe catalogue ${ }^{\star}$
}

\author{
W.T. Manrique ${ }^{1}$, Lu. Lizhi ${ }^{2}$, R. Perdomo ${ }^{3}$, R.C. Podesta ${ }^{1}$, Wang Zezhi ${ }^{2}$, E.L. Actis ${ }^{1}$, Zeng Fanmiao ${ }^{2}$ Zeng Zhifang $^{2}$, \\ E. Alonso ${ }^{1}$, A.M. Pacheco ${ }^{1}$, and G. Bustos ${ }^{1}$ \\ 1 Observatorio Astronomico Felix Aguilar, Benavidez 8175, San Juan, Argentina \\ 2 Beijing Astronomical Observatory, Beijing 100080, China \\ 3 Observatorio Astronomico de La Plata, 1900 La Plata, Argentina
}

Received December 12, 1997; accepted June 4, 1998

\begin{abstract}
According to the cooperation between Beijing, San Juan and La Plata Astronomical Observatories, the photoelectric astrolabe Mark II(PAII) of the Beijing Astronomical Observatory was moved and installed at the San Juan Observatory, Argentina in January, 1992 for observations of the catalogue of stars in the southern hemisphere. The first observing period was from Feb. 23, 1992 to Mar. 31, 1997. Using the data observed in San Juan with the instrument during this period, residuals for 11002 stars are reduced from about 405700 observations of stars over 1532 days. The mean precision of the residuals is $\pm 0.043^{\prime \prime}$. The Second Catalogue of Stars (CPASJ2) has been compiled from double transits at both the eastern and western passages. There are 5241 stars in this catalogue, including 1225 FK5/FK4Supp stars, 794 FK5Ext stars, 1084 SRS stars, 937 CAMC4 stars, 310 GC stars and 891 IMF stars. The mean precisions are $\pm 3.2 \mathrm{~ms}$ and $\pm 0.057^{\prime \prime}$ in right ascension and declination, respectively. The magnitudes of stars are from 2.0 to 11.5 . The declinations are from $-3^{\circ}$ to $-60^{\circ}$. The mean epoch is 1994.9. Finally, systematic corrections of(CPASJ2-FK5) are given.
\end{abstract}

Key words: catalogue — astrometry — reference systems

\section{Introduction}

The photoelectric astrolabes Mark II were built at the Nanjing Astronomical Instrument Factory in 1974. These instruments are used for determinations of astronomical time, latitude, and star positions as well as for research in astrogeodynamics.

\footnotetext{
* The catalogue is only available in electronic form at the CDS via anonymous ftp to cdsarc.u-strasbg.fr (130.79.128.5) or via http://cdsweb.u-strasbg.fr/Abstract.html
}

The photoelectric astrolabe Mark II No. 2 was installed at the Shahe Station of the Beijing Astronomical Observatory at the end of 1975 and was put into operation since Mar. 1976 (Luo 1979). The aperture of the primary mirror is $200 \mathrm{~mm}$ and that of the secondary mirror $49 \mathrm{~mm}$. The equivalent focal length is $2400 \mathrm{~mm}$. The zenith distance of observation is $30^{\circ}$. A vacuum telescope tube and two reflecting mirrors with a stable angle are used. This optical system of the astrolabe is quite stable.

Before modernization, the astrolabe was a semiautomatic instrument with a limiting magnitude of only 7.0. Since 1987, the astronomers of the Beijing Astronomical Observatory began to improve the instrument. Automatic observation with the instrument started in 1988. A new method of photon-counting was used for data processing in 1990, and the limiting magnitude of observation correspondingly increased to 11.5 .

Since 1980, several general catalogues (Zhu et al. 1981; Working Group of GCPA 1983; Working Group of CGSC 1991; Lu 1991) of stars have been compiled using the data observed with the different types of Chinese photoelectric astrolabes.

The Fundamental Reference System of Stars project is important for astrometry. Although Hipparcos has made remarkable achievements, there are many things to do after Hipparcos (Clauzet et al. 1990; Kovalevsky 1991), such as: the improvement of fundamental reference system of stars; the extension of fundamental catalogue to faint stars; the maintenance of Hipparcos reference system; the extension of the Hipparcos catalogue. Two types of ground-based instruments can do these works: the photoelectric meridian circles and the photoelectric astrolabes which both give star positions with a rms precision of the order of $0.1^{\prime \prime}$.

During the IAU 20th General Assembly at Baltimore in 1988, the Working Group "Astrolabe", established by Commission 8 had decided to undertake the compilation of a New General Astrolabe Catalogue (NCGA). 
Table 1. The relation between residual and magnitude

\begin{tabular}{|c|cccccccccc|}
\hline $\bar{M}_{v}$ & 2.2 & 3.1 & 4.0 & 5.0 & 5.9 & 7.0 & 8.0 & 9.0 & 9.9 & 10.7 \\
$N$ & 47 & 143 & 339 & 786 & 1086 & 710 & 1056 & 1683 & 961 & 162 \\
$\bar{V}_{\mathrm{m}}\left( \pm 0.01^{\prime \prime}\right)$ & -1.1 & -0.6 & -0.1 & -0.1 & 0.8 & 0.3 & 0.4 & 0.1 & -0.1 & 1.8 \\
$m_{\bar{V}_{\mathrm{m}}}\left( \pm 0.01^{\prime \prime}\right)$ & 0.9. & 0.4 & 0.3 & 0.2 & 0.2 & 0.4 & 0.4 & 0.3 & 0.5 & 1.3 \\
\hline
\end{tabular}

Astronomers from China and Argentina were interested in the project. According to the cooperation between Beijing, San Juan, and La Plata Astronomical Observatories, the photoelectric astrolabe Mark II No. 2 of the Beijing Astronomical Observatory was moved and installed at San Juan Observatory, Argentina in January 1992 for observations of the catalogue of stars in the southern hemisphere.

Using the data observed with the instrument from Feb. 1992 to Feb. 1995, the First San Juan Catalogue was published ( $\mathrm{Lu}$ et al. 1996). In this paper, we present all results obtained during the first stage of the cooperation from Feb. 1992 to Mar. 1997. The second stage started in April, 1997, and will be continued until Mar. 2002. We hope that these observations will make a contribution to new fundamental reference system of stars.

\section{The observation program}

The purpose of observations with PA II in San Juan is the improvement of the fundamental catalogue in southern hemisphere. The list of stars is selected from:

(a) FK5 fundamental stars (592 stars, $2 \mathrm{mag}-8 \mathrm{mag}$ ).

(b) FK4 Supp stars (657 stars, 2 mag $-8 \mathrm{mag}$ ).

(c) FK5 Ext stars (822 stars, 6 mag - 9.5 mag).

(d) SRS stars (1372 stars, $6 \mathrm{mag}-9.5 \mathrm{mag}$ ).

(e) IMF stars, that is Intermediate Fundamental Stars (1079 stars, 9 mag - $11.5 \mathrm{mag}$ ).

(f) CAMC4 stars (1080 stars, $9 \mathrm{mag}-11.5 \mathrm{mag}$ ).

(g) GC stars (354, 2 mag - $6 \mathrm{mag})$.

(h) Radio stars (82 stars, $2 \mathrm{mag}-11.5 \mathrm{mag}$ ).

(i) Some solar system objects.

There are about 6038 stars in the list. The magnitudes are from 2.0 to 11.5 . The declinations are from $-3^{\circ}$ to $-60^{\circ}$, The positions of FK5, FK4 Supp., and FK5 Ext stars in J2000.0 are taken from FK5 Fundamental Catalogue and FK5 Extended Fundamental Catalogue provided by CDS. The positions of the Intermediate Fundamental Stars were provided by Dr. Thomas E. Corbin at U.S. Naval Observatory. These stars are selected from the Hipparcos Input Catalogue provided by CDS. The positions of the radio stars were taken from the literature (Walter 1990). The positions of GC stars were from the Chinese Geodetical Stars Catalogue (CGSC). The others were from CAMC4 and SRS catalogues. All the positions of stars have been reduced to the epoch and equinox J2000.0.
Table 2. The relation between residual and spectral type

\begin{tabular}{|c|cccccc|}
\hline Sp. & $\mathrm{B}$ & $\mathrm{A}$ & $\mathrm{F}$ & $\mathrm{G}$ & $\mathrm{K}$ & $\mathrm{M}$ \\
$N$ & 691 & 1027 & 1144 & 1428 & 2390 & 247 \\
$\bar{V}_{\mathrm{s}}\left(0^{\prime \prime} 01^{\prime \prime}\right)$ & -1.0 & 0.3 & 0.2 & -0.3 & 0.8 & 0.7 \\
$m_{\bar{V}_{\mathrm{s}}}\left(0.01^{\prime \prime}\right)$ & 0.3 & 0.3 & 0.3 & 0.3 & 0.2 & 0.5 \\
\hline
\end{tabular}

\section{Reduction of the data}

\subsection{The reduction of star residuals}

The fundamental equation of observation is

$15 X \cos \varphi_{0} \sin A+Y \cos A-Z+\delta h=0$,

where

$\varphi_{0}-$ the adopted value of the latitude at the site of the instrument;

$A$ - the azimuth of the observation, measured eastwards from the north;

$X$ - the observed clock correction;

$Y$ - the correction of latitude;

$Z$ - the correction of instrumental zenith distance;

$\delta h-$ a known term which is related to the position of the instrument and systematic errors in the observation.

As the Eqs. (1) are not strictly verified in the least square method of resolution, each of them gives a residual $\mathrm{v}$ which can be computed as:

$v=15 X \cos \varphi_{0} \sin A+Y \cos A-Z+\delta h$.

There are 384 stars in 12 fundamental groups. The corrections of astronomical time, latitude, zenith distance and residuals are obtained by observing the stars of fundamental groups and by solving for each fundamental group the system of Eqs. (2). The residuals of the catalogue stars, observed at the same epoch are computed applying the corrections of astronomical time, latitude and zenith distance found from fundamental groups observations. Then, the mean values of the star residuals are computed by weighted means. The weight $P$ 's are computed from the formula:

$P=\frac{0.1}{\sigma^{2}}$

where $\sigma$ is the precision of a single star observation in the reference group of stars. 
Table 3. The group corrections $\mathrm{d} t, \mathrm{~d} \varphi$, and $\mathrm{d} z$

\begin{tabular}{|c|rrrrrrrrrrrr|r|}
\hline Group & 0 & 2 & 4 & 6 & 8 & 10 & 12 & 14 & 16 & 18 & 20 & $\begin{array}{r}22 \\
\text { closing } \\
\text { error }\end{array}$ \\
\hline $\mathrm{d} t\left(0.0001^{\mathrm{s}}\right)$ & -53 & 04 & 22 & 29 & 32 & 09 & -25 & -29 & -17 & 01 & 13 & 14 & 13 \\
$\sigma_{\mathrm{t}}\left( \pm 0.0001^{\mathrm{s}}\right)$ & 8 & 8 & 8 & 8 & 8 & 8 & 8 & 8 & 8 & 8 & 8 & 8 & 28 \\
$\mathrm{~d} \varphi\left(0.001^{\prime \prime}\right)$ & 28 & 20 & -23 & -06 & 46 & 10 & -17 & 07 & 20 & 08 & -66 & -28 & -59 \\
$\sigma_{\mathrm{d} \varphi\left( \pm 0.001^{\prime \prime}\right)}(2$ & 11 & 11 & 11 & 10 & 10 & 10 & 11 & 11 & 12 & 12 & 12 & 39 \\
$\mathrm{~d} z\left(0.001^{\prime \prime}\right)$ & -01 & -23 & -62 & -30 & 35 & 05 & -10 & 43 & 37 & 08 & 03 & -06 & 110 \\
$\sigma_{\mathrm{d} z}\left( \pm 0.001^{\prime \prime}\right)$ & 9 & 8 & 8 & 8 & 8 & 8 & 8 & 8 & 8 & 8 & 9 & 9 & 29 \\
\hline
\end{tabular}

Table 4a. The frequency distribution of $\Delta \alpha$

\begin{tabular}{|c|c|cccccc|}
\hline & $|\Delta \alpha|(\mathrm{ms})$ & $00-10$ & $10-20$ & $20-30$ & $30-40$ & $40-50$ & $>50$ \\
\hline FK5 & $N$ & 419 & 136 & 21 & 3 & 2 & 0 \\
& $\%$ & 72.1 & 23.4 & 3.6 & 0.5 & 0.3 & 0.0 \\
\hline FK4 Supp. & $N$ & 419 & 166 & 48 & 6 & 3 & 2 \\
& $\%$ & 65.1 & 25.8 & 7.5 & 0.9 & 0.5 & 0.3 \\
\hline FK5 Ext. & $N$ & 382 & 239 & 112 & 34 & 16 & 11 \\
& $\%$ & 48.1 & 30.1 & 14.1 & 4.3 & 2.0 & 1.4 \\
\hline CGSC & $N$ & 179 & 89 & 27 & 7 & 2 & 6 \\
& $\%$ & 57.7 & 28.7 & 8.7 & 2.3 & 0.6 & 1.9 \\
\hline CAMC4 & $N$ & 463 & 257 & 129 & 44 & 23 & 21 \\
& $\%$ & 49.4 & 27.4 & 13.8 & 4.7 & 2.5 & 2.2 \\
\hline SRS & $N$ & 395 & 291 & 154 & 102 & 43 & 99 \\
& $\%$ & 36.4 & 26.8 & 14.2 & 9.4 & 4.0 & 9.1 \\
\hline IMF & $N$ & 240 & 214 & 142 & 91 & 71 & 133 \\
& $\%$ & 26.9 & 24.0 & 15.9 & 10.2 & 8.0 & 14.9 \\
\hline
\end{tabular}

Table $4 \mathbf{b}$. The frequency distribution of $\Delta \delta$

\begin{tabular}{|c|c|cccccc|}
\hline & $|\Delta \delta|\left(0^{\prime \prime} 01\right)$ & $00-15$ & $15-30$ & $30-45$ & $45-60$ & $60-75$ & $>75$ \\
\hline FK5 & $N$ & 298 & 70 & 8 & 2 & 1 & 0 \\
& $\%$ & 78.6 & 18.5 & 2.1 & 0.5 & 0.3 & 0.0 \\
\hline FK4 Supp. & $N$ & 252 & 127 & 24 & 4 & 2 & 0 \\
& $\%$ & 61.6 & 31.1 & 5.9 & 1.0 & 0.5 & 0.0 \\
\hline FK5 Ext. & $N$ & 266 & 164 & 70 & 15 & 6 & 4 \\
& $\%$ & 50.7 & 31.2 & 13.3 & 2.9 & 1.1 & 0.8 \\
\hline CGSC & $N$ & 161 & 72 & 37 & 5 & 7 & 3 \\
& $\%$ & 56.5 & 25.3 & 13.0 & 1.8 & 2.5 & 1.1 \\
\hline CAMC4 & $N$ & 250 & 144 & 71 & 27 & 20 & 12 \\
& $\%$ & 47.7 & 27.5 & 13.5 & 5.2 & 3.8 & 2.3 \\
\hline SRS & $N$ & 339 & 240 & 126 & 80 & 24 & 24 \\
& $\%$ & 40.7 & 28.8 & 15.1 & 9.6 & 2.9 & 2.9 \\
\hline IMF & $N$ & 186 & 147 & 103 & 54 & 36 & 55 \\
& $\%$ & 32.0 & 25.3 & 17.7 & 9.3 & 6.2 & 9.5 \\
\hline
\end{tabular}

\subsection{The reduction of position corrections of stars}

Assumed that $V_{\mathrm{e}}$ and $V_{\mathrm{w}}$ are the residuals reduced to the mean instrumental system and considering the instrumental system errors at both eastern and western transits, we obtain the equation defining the position corrections ( $\mathrm{Lu}$ et al. 1980):

$\Delta \alpha=\frac{V_{\mathrm{e}}-V_{\mathrm{w}}}{30 \cos \varphi_{0}|\sin A|}+\xi$,

and

$\Delta \delta=-\frac{V_{\mathrm{e}}+V_{\mathrm{w}}-2 K}{2 \cos q}+l \cos \delta$, where $q$ is the parallactic angle of the star when it transits the almucantar of the astrolabe.

The term $2 K$ can be computed from

$K=\frac{1}{2}\left(V_{\mathrm{e}}+V_{\mathrm{w}}\right)_{q=90^{\circ}}$.

In fact only the stars with $|\cos q|<0.2$ (in this catalogue there are 736 such stars) are used to computed $2 K$. The weighted mean value of this term is:

$2 K=0.018^{\prime \prime} \pm 0.003^{\prime \prime}$ 
Table 5a. The systematic corrections (CPASJ2-FK5) $\Delta \alpha_{\alpha}$ and $\Delta \delta_{\alpha}$

\begin{tabular}{|c|rrrrrrrrrrrr|}
\hline$\alpha^{\mathrm{h}}$ & 0 & 1 & 2 & 3 & 4 & 5 & 6 & 7 & 8 & 9 & 10 & 11 \\
\hline$\Delta \alpha_{\alpha}\left(0.0001^{\mathrm{s}}\right)$ & -16 & -14 & -22 & -26 & -8 & 30 & 66 & 73 & 46 & 4 & -21 & -16 \\
$\Delta \delta_{\alpha}\left(0.001^{\prime \prime}\right)$ & 6 & 6 & 2 & -1 & 2 & 13 & 27 & 37 & 36 & 23 & 2 & -18 \\
\hline \hline$\alpha^{\mathrm{h}}$ & 12 & 13 & 14 & 15 & 16 & 17 & 18 & 19 & 20 & 21 & 22 & 23 \\
\hline$\Delta \alpha_{\alpha}\left(0.0001^{\mathrm{s}}\right)$ & 4 & 15 & 3 & -19 & -29 & -18 & 4 & 15 & 4 & -17 & -30 & -27 \\
$\Delta \delta_{\alpha}\left(0.001^{\prime \prime}\right)$ & -29 & -26 & -14 & 1 & 10 & 7 & -4 & -17 & -25 & -23 & -14 & -2 \\
\hline
\end{tabular}

Table 5b. The systematic corrections (CPASJ2-FK5) $\Delta \alpha_{\delta}$ and $\Delta \delta_{\delta}$

\begin{tabular}{|c|c|c|c|c|c|c|c|c|c|c|c|c|}
\hline$\delta^{\circ}$ & -2.5 & -5.0 & -7.5 & -10.0 & -12.5 & -15.0 & -17.5 & -20.0 & -22.5 & -25.0 & -27.5 & -30.0 \\
\hline$\Delta \alpha_{\delta}\left(0.0001^{\mathrm{s}}\right)$ & 5 & 39 & 8 & 5 & 26 & 41 & 35 & $\overline{9}$ & -22 & $\overline{-44}$ & $\overline{-46}$ & $\overline{-30}$ \\
\hline$\Delta \delta_{\delta}\left(0.001^{\prime \prime}\right)$ & -64 & -50 & -38 & -27 & -17 & -9 & -1 & 5 & 10 & 14 & & \\
\hline$\overline{\delta^{\circ}}$ & $\overline{-32.5}$ & $\overline{-35.0}$ & -37.5 & $\overline{-240.0}$ & -42.5 & -45.0 & -47.5 & $\overline{-50.0}$ & -52.5 & $\overline{-55.0}$ & -57.5 & $\overline{-60.0}$ \\
\hline$\Delta \alpha_{\delta}\left(0.0001^{\mathrm{s}}\right)$ & $\overline{-6}$ & 15 & 20 & $\overline{7}$ & -20 & $\overline{-49}$ & -65 & -56 & -20 & 30 & 68 & 51 \\
\hline$\Delta \delta_{\delta}\left(0.001^{\prime \prime}\right)$ & & & & & & & 12 & 9 & 5 & 1 & -2 & -6 \\
\hline
\end{tabular}

\section{Results}

From Feb. 23, 1992 to Mar. 31, 1997, there are about 405700 observations of stars over 1532 days. From these data, 11002 residuals are obtained with a mean precision of $\pm 0.043^{\prime \prime} .5241$ position corrections of stars have been obtained using Eqs. (4) and (5) from double transits of these stars at both their eastern and western passages with average precisions $\pm 3.2 \mathrm{~ms}$ and $\pm 0.057^{\prime \prime}$ in right ascension and declination, respectively. The unknown terms $\xi$ and $l \cos \delta$ included in the computed $\Delta \alpha$ and $\Delta \delta$ are neglected. The $\Delta \delta$ for the stars with $|\cos q|<0.3$ are not computed.

\subsection{The relation between residual and magnitude}

The mean values of residuals of stars by weighted mean according to the precision of residuals and magnitudes are given in Table 1 . In Table $1, \bar{M}_{v}$ is the mean values of the observed magnitudes, $N$ is the number of observed stars, $\bar{V}_{\mathrm{m}}$ and $m_{\bar{V}_{\mathrm{m}}}$ are the mean value of residuals and its accuracy.

\subsection{The relation between residuals and spectral types}

The mean values of residuals of stars obtained by a weighted mean according to the precision of residuals and spectral type are given in Table 2 . In Table $2, N$ is the number of observed stars, $\bar{V}_{\mathrm{s}}$ and $m_{\bar{V}_{\mathrm{s}}}$ are the mean value of residuals and its accuracy.

\subsection{The group corrections}

With the results of PA II from Feb. 1992 to Mar. 1997, The group corrections of $\mathrm{d} t, \mathrm{~d} \varphi$ and $\mathrm{d} z$ are computed by the chain method. The results are given in Table 3.

From Table 3, we can see that the closing error of $\mathrm{d} z$ is very small.

\subsection{The frequency distributions of $\Delta \alpha$ and $\Delta \delta$}

The frequency distribution of $\Delta \alpha$ and $\Delta \delta$ are given in Tables $4 \mathrm{a}$ and $4 \mathrm{~b}$ respectively.

From Tables $4 \mathrm{a}$ and $4 \mathrm{~b}$, we can see that the position corrections for about $95 \%$ of the FK5 stars are less than $20 \mathrm{~ms}$ in $\Delta \alpha$ or $0.30^{\prime \prime}$ in $\Delta \delta$.

\subsection{The systematic corrections}

By the analytic method (Bien 1979; Working Group of GCPA 1983) and adopting the $\Delta \alpha$ and $\Delta \delta$ of FK5/FK4 Supp. stars obtained from the double transits, the systematic corrections of the catalogue of stars (CPASJ2-FK5) are analyzed. The systematic corrections on the right ascension and declination, $\Delta \alpha_{\alpha}, \Delta \delta_{\alpha}, \Delta \alpha_{\delta}, \Delta \delta_{\delta}$ are given in Tables $5 \mathrm{a}$ and $5 \mathrm{~b}$, respectively.

\section{The description of the catalogue}

The catalogue(CPASJ2) will be available at the CDS. In the catalogue, the description of each column is given as follows:

1: No: Number in CPASJ2;

2: FK5: Number in FK5 (recorder: 1 - 581), FK4 Supp. (recorder: 582 - 1225), FK5 Ext. (recorder: $1226-$ 2019), SRS (recorder: 2020-3103), CAMC4 (recorder: 3104-4040), GC (recorder 4041-4350) and Hipparcos (recorder: $4351-5241$ ), respectively.

3: Mag: visual magnitude;

4: Sp: spectral type;

5: $\alpha_{2000}$ : right ascension of reference catalogue for epoch and equinox J2000.0;

6: $\delta_{2000}$ : declination of reference catalogue for epoch and equinox J2000.0; 
7: $\Delta \alpha$ : position correction of right ascension in unit of $0.001^{\mathrm{s}}$

8: $\sigma_{\Delta \alpha}:$ mean error of the correction on right ascension in unit of $0.001^{\mathrm{s}}$;

9: $\Delta \delta$ : position correction of declination in unit of $0.01^{\prime \prime}$;

10: $\sigma_{\Delta \delta}:$ mean error of the corrections on declination in unit of $0.01^{\prime \prime}$;

11: $N_{\mathrm{e}}$ : number of observations in the eastern transit;

12: $N_{\mathrm{w}}$ : number of observations in the western transit;

13: Epoch: Mean epoch of observations.

Acknowledgements. The authors wish to thank the CONICET of Argentina, Jan Juan and La Plata Universities, the State Sciences and Technology Commission of China, Chinese Academy of Sciences, National Natural Science Foundation of China, Chinese Astronomy Commission for their support for the project.

\section{References}

Carlsberg Meridian Catalogue in La Palma, 1989

Bien R. Veroff, 1978, Astron. Rechen-Institut, Heidelberg, No. 29

Clauzet L.B.F., Benevides Soares P., et al., 1990, Guidelines for the compilation of a New Astrolabes Catalogue

Kovalevsky J., 1991, Astrophys. Space Sci. 177, 457

Walter H.G., et al., 1990, A\&AS 86, 357

Lu Lizhi, 1991, Publ. Beijing Astron. Obs. 17, 1

Luo Dingjiang, et al., 1979, Publ. Beijing Astron. Obs. 1, 56

Working Group of GCPA, 1983, Acta Astron. Sin. 24, 267

Working Group of CGSC, 1991, Publ. House of Surveying and Mapping, Beijing

Zhu Yuanxian, Lu Lizhi, et al., 1981, Publ. Beijing Astron. Obs. 1,1

Lu Lizhi, Luo Dingjiang, et al., 1980, Acta Astron. Sin. 22, 305

Lu Lizhi, Manrique W.T., Podesta R., et al., 1996, A\&AS 118, 1 